\title{
Two Cases of Vibrio Infection and Sepsis in the Delaware Estuary
}

René Daniel, MD

Center for Human Virology, Division of Infectious Diseases and Environmental Medicine, Department of Medicine, Thomas Jefferson University, 1020 Locust Street, Philadelphia, PA 19107, USA,

Rene.Daniel@jefferson.edu

Joseph Yoo, MD

Thomas Jefferson University Hospital, joseph.yoo@jefferson.edu

Roberto Fratamico, MD

Thomas Jefferson University, roberto.fratamico@jefferson.edu

Robert Measley, MD

Thomas Jefferson University, robert.measley@jefferson.edu

Follow this and additional works at: https://jdc.jefferson.edu/tmf

Part of the Infectious Disease Commons, and the Internal Medicine Commons

Let us know how access to this document benefits you

\section{Recommended Citation}

Daniel, MD, René; Yoo, MD, Joseph; Fratamico, MD, Roberto; and Measley, MD, Robert (2016) "Two Cases of Vibrio Infection and Sepsis in the Delaware Estuary," The Medicine Forum: Vol. 17, Article 12.

DOI: https://doi.org/10.29046/TMF.017.1.013

Available at: https://jdc.jefferson.edu/tmf/vol17/iss1/12

This Article is brought to you for free and open access by the Jefferson Digital Commons. The Jefferson Digital Commons is a service of Thomas Jefferson University's Center for Teaching and Learning (CTL). The Commons is a showcase for Jefferson books and journals, peer-reviewed scholarly publications, unique historical collections from the University archives, and teaching tools. The Jefferson Digital Commons allows researchers and interested readers anywhere in the world to learn about and keep up to date with Jefferson scholarship. This article has been accepted for inclusion in The Medicine Forum by an authorized administrator of the Jefferson Digital Commons. For more information, please contact: JeffersonDigitalCommons@jefferson.edu. 


\title{
Two Cases of Vibrio Infection and Sepsis in the Delaware Estuary
}

\author{
Rene Daniel, MD, Joseph Yoo, MD, Roberto Fratamico, MD, and Robert Measley, MD
}

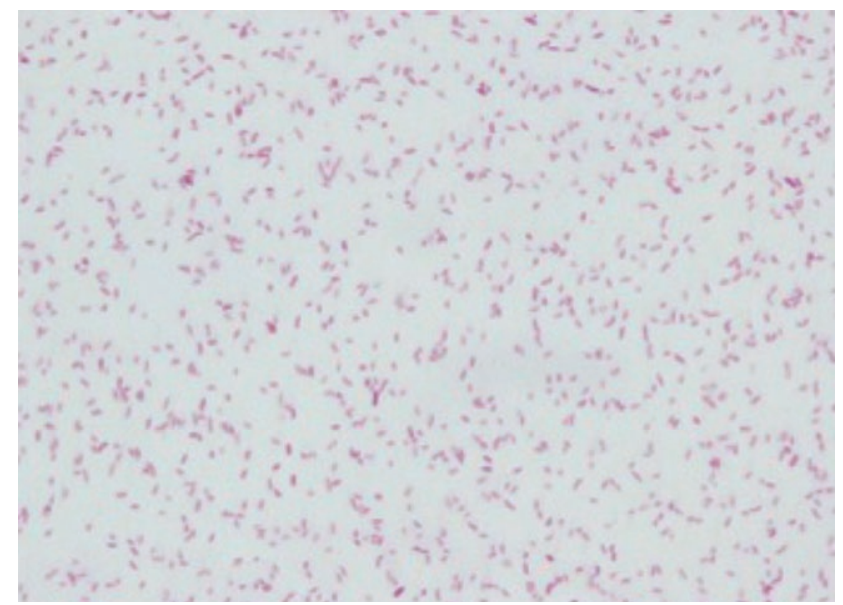

Figure 1. Gram stain of Vibrio vulnificus culture as prepared by the Department of Microbiology of the Wilmington VA Medical Center; Wilmington, Delaware.

\section{INTRODUCTION}

Vibrio is a genus of Gram-negative motile rods. Several species are pathogenic, including Vibrio vulnificus and Vibrio parahaemolyticus. The interest in this genus is due to its extreme virulence and often fatal outcomes. In healthy patients, these infections are typically self-limited; however, in immunocompromised and cirrhotic patients, infections can result in bacteremia, which has a high fatality rate ${ }^{1}$. Both pathogens may cause acute gastroenteritis, necrotizing wound infections, and if severe, subsequent bacteremia. In this report, we present two cases of Vibrio infections recently identified in the Delaware River Estuary that resulted in severe sepsis and, in one case, death.

\section{CASE PRESENTATION}

\section{Case 1}

A 62-year-old male with a past medical history of Hepatitis $\mathrm{C}$ cirrhosis, diabetes mellitus, and chronic venous stasis presented with subjective fevers and lateral left foot and hallux pain. Over the next few hours, the left leg became erythematous with increasing pain accompanied by vomiting. On interview, the patient stated that he went crabbing two days prior to admission without injury.

Vitals on presentation were: temperature 99.0 degrees Fahrenheit, blood pressure 118/85 mm Hg, heart rate 92 beats/minute (BPM), respiratory rate 22 breaths/minute. Physical exam revealed swelling and erythema below the left knee with scattered areas of purple discoloration along the medial forefoot and no crepitus. Pedal pulses were present bilaterally via Doppler. During the next two hours, the purple lesions on the medial aspect of the foot doubled in size and developed central dark greenish discoloration.

\section{Case 2}

A 66-year-old male with a past medical history of Hepatitis $\mathrm{C}$ cirrhosis, alcohol abuse, and atrial fibrillation on warfarin presented with worsening dyspnea for two days. His initial symptoms were abdominal distention followed by productive cough which started few days prior to his hospital admission. On the morning of admission, he noted chills and pleuritic left-sided chest pain.

On presentation, temperature was 101.6 degrees Fahrenheit with a blood pressure of $69 / 49 \mathrm{~mm} \mathrm{Hg}$. He was noted to be in atrial fibrillation with a rapid ventricular response to 175 BPM. Physical exam was notable for an irregularly, irregular heart rate and no jugular venous distention. Lung exam revealed bilateral crackles and use of accessory muscles. Chest x-ray showed new right lower lung and left lower lung opacities consistent with consolidation and a large left-sided pleural effusion with moderate pulmonary edema.

\section{DIFFERENTIAL DIAGNOSIS}

For case 1, the differentials included cellulitis or necrotizing fasciitis from Staphylococcal or Streptococcal species or from atypical organisms found in brackish water such as Aeromonas hydrophila, Pseudomonas aeruginosa and Vibrio species. Limb ischemia was also considered in the differential.

For case 2, the differential included community acquired pneumonia such as from Streptococccus pneumoniae or Legionella pneumophila, aspiration pneumonitis/ pneumonia, and spontaneous bacterial peritonitis.

\section{OUTCOME AND FOLLOW-UP}

\section{Case 1}

Upon admission, a concern for necrotizing fasciitis was raised. Given his exposure to brackish water, a Vibrio infection was suspected. The patient was started on ceftriaxone, doxycycline, and levofloxacin as well as clindamycin for a potential anaerobic infection. His lactate level was $6.6 \mathrm{mg} / \mathrm{dL}$, consistent with severe sepsis. He was transferred to a tertiary care hospital for surgical debridement. Blood cultures grew Vibrio vulnificus on the 


\section{The Medicine Forum, Vol. 17 [2016], Art. 12}

following day (Figure 1). He developed extensive ulcerations of his left leg and underwent surgical debridement. Subsequently he was discharged home. Three months later, he continued to be followed in a wound clinic.

\section{Case 2}

The patient was admitted to the intensive care unit with respiratory failure and septic shock. He required intubation and multiple agents for vasopressor support. Antibiotics were broadened from levofloxacin to vancomycin, piperacillin-tazobactam, azithromycin, and tobramycin. Amiodarone was initiated for rate control of atrial fibrillation. The results of a bedside paracentesis were inconsistent with spontaneous bacterial peritonitis. Blood cultures grew Gram-negative rods in less than 12 hours, at which point piperacillin-tazobactam was switched to meropenem. Unfortunately the patient developed multi-organ failure and expired on comfort care. Blood cultures speciated to Vibrio parahaemolyticus and upon family inquiry it was revealed that he had had a seafood meal days prior to the admission.

\section{DISCUSSION}

Vibrio vulnificus naturally occurs in warm seas (> 20 degrees Celsius) and brackish waters worldwide and accumulates in shellfish ${ }^{1}$. Infections in U.S. coastal regions are relatively rare, with some spikes occurring during hurricane seasons. Reports from the Florida Department of Health highlighted 42 cases of Vibrio vulnificus in 2015, with 13 fatalities, which is the highest number since $2008^{2}$. Rare cases with other manifestations have been described, such as $V$. vulnificus endophthalmitis ${ }^{3}$. Risk factors are crucial for development of $V$. vulnificus sepsis, since less than $5 \%$ of reported cases occurred in individuals without risk factors ${ }^{1}$. These risk factors include chronic liver disease, especially alcoholic and/or hepatitis B or C cirrhosis, immunodeficiencies including those due to chemotherapy or steroid use, AIDS, end stage renal disease, diabetes mellitus, and hematologic disorders associated with high blood iron levels. This is due to the fact that $V$. vulnificus requires iron for its growth and grows rapidly when transferrin saturation exceeds $70 \%$. The treatment involves prompt antibiotic administration, and when soft tissue infection occurs, surgical debridement is essential. The Centers for Disease Control and Prevention recommends treatment with doxycycline and a third generation cephalosporin which are thought to be synergistic in $V$. vulnificus infection ${ }^{5}$. Alternatively, single agent treatment with a fluoroquinolone is thought to be equally effective ${ }^{6}$. Unfortunately, since $V$. vulnificus infections are rare and vary in presentation, it is often under recognized ${ }^{7.8}$. In Case 1 , V. vulnificus appears to have been due to skin entry, although a wound entry site was not identified. Given the patient's cirrhosis and the report of crabbing in brackish water two days prior, the medical team was able to make a prompt diagnosis based on a high index of suspicion. As a result, appropriate antibiotic therapy that went beyond the traditional default broad spectrum coverage for hospitalized patients was initiated and prompt transfer for debridement occurred.

Vibrio parahaemolyticus infection shares many features with $V$. vulnificus infection although its course is usually milder. An average of 215 culture-confirmed cases, 30 hospitalizations, and 1-2 deaths are reported each year

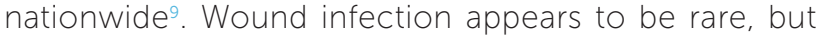
several cases were described during Hurricane Katrina, and two had fatal outcomes ${ }^{10}$. As with V. vulnificus, sepsis can occur and may have a fatal outcome, with risk factors similar to those of $V$. vulnificus ${ }^{9}$. The symptoms of $V$. parahaemolyticus infection usually include severe watery diarrhea, nausea, vomiting, abdominal cramps, and occasional fever'. Infection is usually self-limited and symptoms resolve in 72 hours. Skin manifestations occur when the entry is a skin wound ${ }^{9}$. Our case of $V$. parahaemolyticus infection was challenging to diagnose since the presentation was that of a pneumonia, and only afterwards was the history of shellfish ingestion obtained.

\section{KEY POINTS}

Both cases emphasize the importance of having a high index of suspicion for Vibrio infections during the summer months in coastal regions given the rapid progression and high mortality of infections. High risk individuals include those who are immunocompromised or have cirrhosis, elevated blood iron levels, or have chronic inflammatory conditions. Prompt initiation of appropriate antibiotics is the key to successful treatment.

\section{REFERENCES}

1. Horseman MA, Surani S. A comprehensive review of vibrio vulnificus: an important cause of severe sepsis and skin and soft-tissue infection. Int J Infect Dis. 2011 Mar;15(3):e157-66.

2. Herriman, Robert. "Florida Report Most Vibrio Vulnificus Cases in Years." Outbreak News Today, 14 Nov. 2015. Web. <http://outbreaknewstoday.com/ florida-reports-most-vibrio-vulnificus-cases-in-years-24498/>

3. Jung SI, Shin DH, Park KH, Shin JH, Seo MS. Vibrio vulnificus endophthalmitis occurring after ingestion of raw seafood. J Infect. 2005 Dec;51(5):e281-3.

4. Hollis DG, Weaver RE, Baker CN, Thornsberry C. Halofilic Vibrio species isolated from blood cultures. J Clin Microbiol. 1976 Apr;3(4):425

5. Chuang YC, Liu JW, KoWC, Lin KY, Wu JJ, Huang KY. In vitro synergism between cefotaxime and minocycline against Vibrio vulnificus. Antimicrob Agents Chemother 1997:41:2214-7.

6. "Vibrio Vulnificus." Centers for Disease Control and Prevention, 21 Oct. 2013 Web. <http://www.cdc.gov/vibrio/vibriov.html>.

7. You JS, Kim S, Park I, Kim S. Vibrio vulnificus sepsis misdiagnosed as simple vein thrombosis. Am J Emerg Med. 2012 Nov;30(9):2098.e5-6

8. Ryu HH, Lee JY, Yun NR, Kim DM. Necrotizing soft tissue infection with gas formation caused by Vibrio vulnificus and misdiagnosed as Pseudomonas aeruginosa. Am J Emerg Med. 2013 Feb;31(2):464.e5-8.

9. "Vibrio Parahemolyticus." Centers for Disease Control and Prevention, 21 Oct. 2013. Web. <http://www.cdc.gov/vibrio/vibriop.html>

10. "Vibrio Illnesses After Hurricane Katrina --- Multiple States, August--September 2005." MMWR. Centers for Disease Control and Prevention, 14 Sept. 2005 Web. <http://www.cdc.gov/mmwr/preview/mmwrhtml/mm54d914a1.htm> 